\title{
Are prehospital airway management resources compatible with difficult airway algorithms? A nationwide cross-sectional study of helicopter emergency medical services in Japan
}

\author{
Yuko Ono $^{1,2} \cdot$ Kazuaki Shinohara $^{2} \cdot$ Aya Goto $^{3} \cdot$ Tetsuhiro Yano $^{1} \cdot$ Lubna Sato $^{1} \cdot$ \\ Hiroyuki Miyazaki ${ }^{1} \cdot$ Jiro Shimada $^{1} \cdot$ Choichiro Tase $^{1}$
}

Received: 28 July 2015 / Accepted: 13 December 2015 / Published online: 29 December 2015

(C) The Author(s) 2015. This article is published with open access at Springerlink.com

\begin{abstract}
Purpose Immediate access to the equipment required for difficult airway management (DAM) is vital. However, in Japan, data are scarce regarding the availability of DAM resources in prehospital settings. The purpose of this study was to determine whether Japanese helicopter emergency medical services (HEMS) are adequately equipped to comply with the DAM algorithms of Japanese and American professional anesthesiology societies.

Methods This nationwide cross-sectional study was conducted in May 2015. Base hospitals of HEMS were mailed a questionnaire about their airway management equipment and back-up personnel. Outcome measures were (1) call for help, (2) supraglottic airway device (SGA) insertion, (3) verification of tube placement using capnometry, and (4) the establishment of surgical airways, all of which have been endorsed in various airway management guidelines. The criteria defining feasibility were the availability of (1) more than one physician, (2) SGA, (3) capnometry, and (4) a surgical airway device in the prehospital setting.
\end{abstract}

Electronic supplementary material The online version of this article (doi:10.1007/s00540-015-2124-7) contains supplementary material, which is available to authorized users.

Yuko Ono

windmill@fmu.ac.jp

1 Emergency and Critical Care Medical Center, Fukushima Medical University Hospital, 1 Hikarigaoka, Fukushima 960-1295, Japan

2 Department of Anesthesiology, Ohta General Hospital Foundation, Ohta Nishinouchi Hospital, Koriyama, Japan

3 Department of Public Health, School of Medicine, Fukushima Medical University, Fukushima, Japan
Results Of the 45 HEMS base hospitals questioned, 42 $(93.3 \%)$ returned completed questionnaires. A surgical airway was practicable by all HEMS. However, in the prehospital setting, back-up assistance was available in $14.3 \%$, SGA in $16.7 \%$, and capnometry in $66.7 \%$. No HEMS was capable of all four steps.

Conclusion In Japan, compliance with standard airway management algorithms in prehospital settings remains difficult because of the limited availability of alternative ventilation equipment and back-up personnel. Prehospital health care providers need to consider the risks and benefits of performing endotracheal intubation in environments not conducive to the success of this procedure.

Keywords Airway equipment $\cdot$ Supraglottic airway device · Difficult airway · Prehospital endotracheal intubation · Surgical airway equipment

\section{Introduction}

Helicopter emergency medical services (HEMS) have been implemented in Japan since 2001 [1], with recent rapid increases in their use (Fig. 1). Annual HEMS dispatches exceeded 20,000 in 2013 (Fig. 1), and the number continues to rise [data kindly provided by the Japanese Society for Aeromedical Services, and the Emergency Medical Network of Helicopter and Hospital (HEM-Net)]. After the major earthquake in eastern Japan in 2011, HEMS played a crucial role in disaster-stricken areas by providing triage, treatment, emergency care, and transportation [2]. With the rapid growth of HEMS in Japan and the improved response to catastrophes such as earthquakes, prehospital endotracheal intubation (ETI) has become much more common. 
Fig. 1 Growth of helicopter emergency medical services (HEMS) in Japan. The data were provided by the Japanese Society for Aeromedical Services, and the Emergency Medical Network of Helicopter and Hospital (HEM-Net)

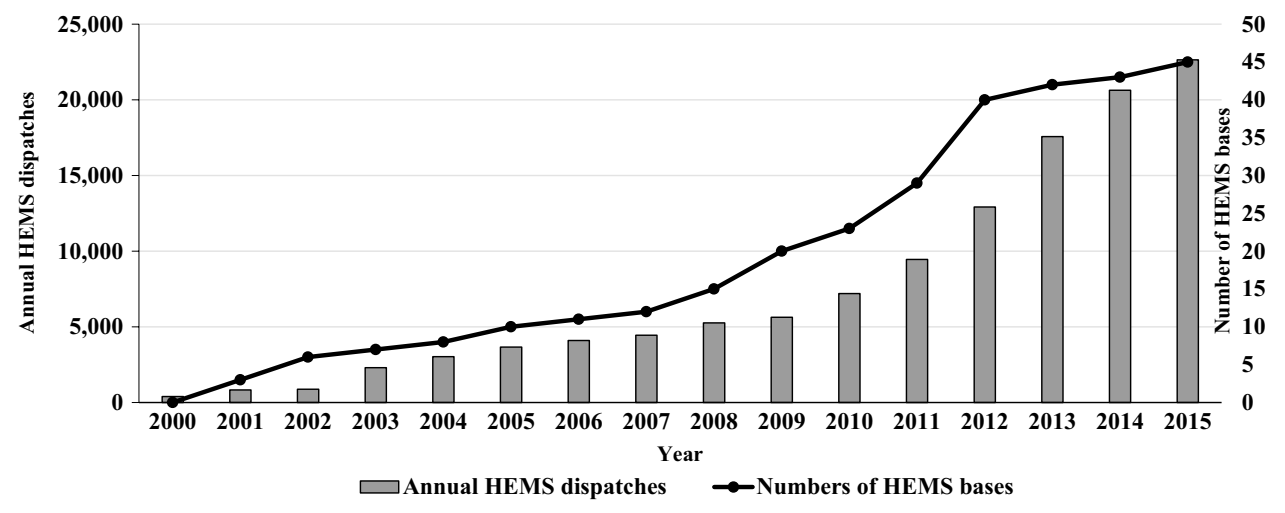

ETI outside the hospital is challenging even for experienced providers. The rate of difficult ETI in prehospital settings ranges from 6.0-17.7\% [3-6], which is much higher than the rate in the hospital operating room [4]. Severe ETIrelated complications, including severe hypoxia, esophageal intubation, aspiration, and cardiac arrest, are likely to occur in association with difficult airway management (DAM) [79]. Although the limited resources of prehospital settings are in part responsible for these difficulties [10], data are scarce regarding the availability of airway equipment, alternative ventilation devices, and drugs and the capabilities of care providers in Japanese prehospital environments.

Airway management algorithms have been advocated by the Japanese Society of Anesthesiologists (JSA) [11], the American Society of Anesthesiologists (ASA) [12], and by the Difficult Airway Society (DAS) [13]. These guidelines underlie the standards and principles that apply to the treatment of a patient with a difficult airway, not only regarding the induction of anesthesia but also for any other situation arising in the emergency department and prehospital setting. Their four key steps are [11-13] (1) call for help if any problems have occurred; if mask ventilation is not adequate, consider (2) supraglottic airway device (SGA) insertion; (3) confirmation of ETI using capnometry; and (4) establish a surgical airway if a 'cannot ventilate, cannot intubate' (CVCI) situation is encountered. The immediate availability of back-up staff and of proper DAM equipment, including SGA, capnometry, and a surgical airway device, is therefore indispensable. The authors of a report based on a national survey carried out in the UK concluded that, regardless of the location, DAM equipment should be consistent with that in the hospital operating room [14]. In fact, several studies have proposed that ETI in the prehospital setting should be performed according to the same standards that apply in the hospital [15-17]. However, whether prehospital airway management resources in Japan are compatible with the standards established in the DAM guidelines [11-13] has not been comprehensively evaluated.

Therefore, by conducting a national survey of HEMS, we sought to determine (1) the availability of airway devices, alternative ventilation, ETI confirmation equipment, drugs, and specialist care providers and (2) whether these resources comply with the JSA, ASA, and DAS airway management algorithms [11-13].

\section{Materials and methods}

\section{Study design and sites}

This cross-sectional study was conducted from May to July 2015. After approval by the institutional review boards of Fukushima Medical University (no. 2276), self-administered questionnaires were mailed to all HEMS base hospitals ( 45 bases in 37 prefectures) registered in HEM-Net. A complete list of these hospitals is available at the HEM-Net home page: http://www.hemnet.jp/english/where/index. html (accessed 22 October 2015).

\section{Survey items}

When selecting items in the questionnaire, we referred to previous studies conducted in other countries and addressing similar (prehospital settings [18-20], emergency departments [21-23]) as well as different (obstetric units [24-26]) settings. We then circulated drafts among survey team members consisting of an epidemiologist, anesthesiologists, and physicians and nurses specializing in emergency medicine to finalize.

These survey items consisted of (1) basic information regarding the numbers of HEMS dispatches in 2014 and hospital beds, and the prehospital availability of the following materials-(2) direct laryngoscope and adjunct equipment (curved blade, straight blade, McCoy laryngoscope, stylet and gum elastic bougie); (3) alternative intubation equipment (rigid video laryngoscope, flexible fiber scope, retrograde intubation kit, and surgical airway equipment); (4) alternative ventilation equipment (SGA, oral and nasal airways); (5) device to confirm ETI (capnometry, esophageal detector); (6) a packaged unit containing the items listed in (2)-(4); and (7) drugs to facilitate ETI and reversal agents (analgesics, sedative, neuromuscular blocking 
agents [NMBAs], sugammadex, flumazenil and naloxone). In addition, information about (8) the prehospital care providers (number of prehospital physicians, nurses and on-the-job trainees) usually on board the emergency helicopters and (9) the board certifications of the prehospital physicians and nurses was obtained. Among these items, in (6)-(8) and in (9), the board certification of nurses had not been included in previous studies [18-26]. However, it was included in this study after discussion and mutual agreement among the authors. An English version of the Japanese questionnaire used in this study is available in the supplementary material. The questionnaire also queried the availability of direct laryngoscopes and alternative ventilation equipment in pediatric sizes. The product name of the rigid video laryngoscopes used was also requested. The contents of the packaging unit were determined according to the airway management guidelines of the JSA [11] and ASA [12]. Packaging was graded as complete, partial, and none. If SGA was available, its inclusion of an intubating laryngeal mask was determined. Surgical airway equipment was categorized as a cricothyroidotomy kit or a set containing a scalpel and hemostat. Board-certified physicians and nurses were defined based on the criteria of the Japanese Medical Specialty Board (http://www.japan-senmon-i.jp/, in Japanese, accessed 22 October 2015) and Japanese Nursing Association (http://nintei.nurse.or.jp/nursing/qualification/cn, in Japanese, accessed 22 October 2015), respectively. HEMS base hospitals that did not respond to the initial survey were sent a repeat mailing.

\section{Outcome measures}

The JSA airway management algorithms [11], ASA DAM guideline [12], and DAS guideline [13] commonly endorse the following four steps in the treatment of an airway(1) call for help if difficulties are encountered; (2) attempt SGA insertion if mask ventilation is not adequate; (3) use of capnometry to confirm correct endotracheal tube placement; and (4) establish a surgical airway if a CVCI situation has occurred. Outcome measures in this study included the feasibility of these four steps in the prehospital settings. 'Call for help' was deemed feasible if more than one physician was usually on board. This is because, in Japan, only physicians are permitted to perform ETI and SGA insertion. On-the-job medical trainees were not regarded as physicians because they could have been stafflevel physicians, junior residents, nurses, or paramedics. 'SGA insertion' and 'confirmation of ETI using capnometry' were presumed possible if the respective devices were carried on board. 'Surgical airway' was deemed practicable if a cricothyroidotomy kit or a scalpel and hemostat were available on board. All outcome measures were defined by mutual consent among the five authors (YO,
KS, AG, JS, and CT), which included three board-certified anesthesiologists.

\section{Statistical analysis}

First, all survey items were evaluated using descriptive statistics. Second, the association between the feasibility of the four steps and annual HEMS dispatches, regions, and foundation date were analyzed using Fisher's exact test. For HEMS dispatches, the values were dichotomized using the median. Regions were divided into east (Hokkaido, Tohoku, Kanto/Koshin, Hokuriku, Tokai) and west (Kinki, Chugoku, Shikoku, North and South Kyushu, Okinawa) according to the classification of the Japanese meteorological agency (http://www.jma.go.jp/jma/indexe. $\mathrm{html}$, accessed 22 October 2015). The foundation date was divided into an early phase (2001-2008) and a late phase (2008-2015). All statistical analyses were performed using IBM SPSS Statistics for Windows, version 21.0 (IBM Corp., Armonk, NY, USA). A $p$ value $<0.05$ was considered to indicate statistical significance.

\section{Results}

Of the 45 HEMS base hospitals, 42 returned the completed questionnaire (response rate $=93.3 \%$ ). None of these hospitals were excluded because of incomplete responses. The median number of annual HEMS dispatches was 447 (interquartile range 366-550); the median number of hospital beds was 653 (interquartile range 579-768). Table 1 summarizes the airway equipment available in Japanese HEMS. Among the HEMS bases that responded, only seven $(16.7 \%)$ had a SGA, five $(11.9 \%)$ of which also carried a pediatric-sized device. Capnometry was available in 28 (66.7 \%) HEMS. All bases possessed a surgical airway device, either a cricothyroidotomy kit (61.9\%) or scalpel and hemostat $(38.1 \%)$. Table 2 lists the drugs available to facilitate ETI in prehospital settings. None of the HEMS had depolarizing NMBAs; $34(81.0 \%)$ had at least one non-depolarizing NMBA, 5 (11.9\%) had sugammadex, and $8(19.0 \%)$ did not have any type of NMBA. Table 3 provides information on the prehospital care providers. Two physicians were usually on board at six (14.3\%) bases. Of 347 attending physicians at all bases, the most common board certification was emergency medicine $(75.8 \%)$, followed by general surgery $(15.6 \%)$. Board-certified anesthesiologists comprised $10.1 \%$ of all prehospital physicians. Figure 2 shows the availability in Japanese HEMS of the DAM resources specified in the JSA, ASA, and DAS algorithms. According to our feasibility definitions, 'surgical airway' was deemed attainable in all bases, 'call for help' in $14.3 \%$, 'SGA insertion' in 
Table 1 Airway equipment at 42 Japanese helicopter emergency medical services (HEMS)

\begin{tabular}{|c|c|}
\hline Equipment item & $N(\%)$ \\
\hline \multicolumn{2}{|l|}{ Direct laryngoscope and adjunct ${ }^{\mathrm{a}}$} \\
\hline Curved laryngoscope blade (Macintosh type) & $42(100)$ \\
\hline Pediatric size & $39(92.9)$ \\
\hline Straight laryngoscope blade (Miller type) & $31(73.8)$ \\
\hline Pediatric size & $31(73.8)$ \\
\hline McCoy laryngoscope & $0(0)$ \\
\hline Stylet & $41(97.6)$ \\
\hline Gum elastic bougie & $14(33.3)$ \\
\hline \multicolumn{2}{|l|}{ Alternative intubation equipment } \\
\hline Rigid video laryngoscope $\mathrm{a}^{\mathrm{a}}$ & $39(92.9)$ \\
\hline Airway scope ${ }^{\circledR}$ & $33(78.6)$ \\
\hline McGRATH MAC ${ }^{\circledR}$ & $12(28.6)$ \\
\hline King Vision ${ }^{\circledR}$ & $1(2.4)$ \\
\hline Airtraq $^{\circledR}$ & $1(2.4)$ \\
\hline Flexible fiber scope & $3(7.1)$ \\
\hline Retrograde intubation kit & $1(2.4)$ \\
\hline Surgical airway equipment & $42(100)$ \\
\hline Cricothyroidotomy kit & $26(61.9)$ \\
\hline Scalpel and hemostat & $16(38.1)$ \\
\hline \multicolumn{2}{|l|}{ Alternative ventilation equipment ${ }^{\mathrm{a}}$} \\
\hline Supraglottic airway device & $7(16.7)$ \\
\hline Pediatric size & $5(11.9)$ \\
\hline Intubating laryngeal mask airway & $2(4.8)$ \\
\hline Oral airway & $21(50.0)$ \\
\hline Pediatric size & $16(38.1)$ \\
\hline Nasal airway & $36(85.7)$ \\
\hline Pediatric size & $8(19.0)$ \\
\hline \multicolumn{2}{|l|}{ Device to confirm endotracheal intubation ${ }^{a}$} \\
\hline Capnometry & $28(66.7)$ \\
\hline Esophageal detector & $7(16.7)$ \\
\hline Any other devices & $4(9.5)$ \\
\hline \multicolumn{2}{|l|}{ Packaging unit containing items $1-4$} \\
\hline Complete packaging & $16(38.1)$ \\
\hline Partial packaging & $15(35.7)$ \\
\hline No packaging & $11(26.2)$ \\
\hline
\end{tabular}

Based on the replies of 42 of the 45 HEMS queried

a HEMS base hospitals may have more than one of the specified equipment items

$16.7 \%$ (11.9\% in pediatric cases), and 'confirmation of ETI using capnometry' in $66.7 \%$. There were no bases in which all steps were deemed achievable in the prehospital setting. Table 4 shows the associations between the feasibility of airway management guidelines and annual dispatches, region, and the foundation dates of the HEMS surveyed. None of the associations were of statistical significance.
Table 2 Drugs that facilitate prehospital endotracheal intubation and reversal agents carried by Japanese helicopter emergency medical services (HEMS)

\begin{tabular}{lc}
\hline Item & $N(\%)$ \\
\hline Analgesics & \\
Fentanyl & \\
Morphine & $13(31.0)$ \\
Ketamine & $16(38.1)$ \\
Pentazocin & $12(28.6)$ \\
Buprenorphine & $25(59.5)$ \\
Lidocaine & $15(35.7)$ \\
Lidocaine spray & $29(69.0)$ \\
Any other analgesic & $6(14.3)$ \\
Sedatives & \\
Midazolam & $0(0)$ \\
Diazepam & \\
Propofol & $39(92.9)$ \\
Thiopental & $38(90.5)$ \\
Haloperidol & $10(23.8)$ \\
Any other sedatives & $4(9.5)$ \\
Neuromuscular blocking agents ${ }^{\mathrm{a}}$ & $2(4.8)$ \\
Rocuronium & $0(0)$ \\
Vecuronium & \\
Pancuronium & $19(45.2)$ \\
Succinylcholine & $18(42.9)$ \\
Any other neuromuscular blocking agents & $0(0)$ \\
Reversal agents & \\
Sugammadex & $0(0)$ \\
Flumazenil & $0(0)$ \\
\hline & \\
Naloxone & $5(11.9)$ \\
& $1(2.4)$ \\
& $1(2.4)$ \\
\hline
\end{tabular}

Based on the replies of 42 of the 45 HEMS queried

${ }^{a}$ HEMS base hospitals may have more than one drug

\section{Discussion}

In Japan, compliance with standard DAM algorithms [1113] in prehospital settings is not currently feasible because of the limited availability of back-up personnel and alternative ventilation equipment. Our study showed that additional assistance and SGA were available in $<20 \%$ of HEMS bases. Although surgical airway was attainable at all base hospitals, no hospital was able to attain all of the steps. Of note was that only one in ten prehospital physicians were board-certified anesthesiologists. These findings suggest that, in their current form, prehospital settings in Japan do not allow safe ETI. All care providers who participate in prehospital airway management should be aware of the limited human and equipment resources encountered under current working conditions. If the patient is expected to have a difficult airway, ETI should not be attempted in 
Table 3 On-board medical members in Japanese helicopter emergency medical services (HEMS) ${ }^{\mathrm{a}}$

\begin{tabular}{ll}
\hline Item & $N(\%)$ \\
\hline On-board staff members & $N=42$ \\
Two physicians and one nurse & $6(14.3)$ \\
One physician and one nurse & $20(47.6)$ \\
One physician, one nurse, and one on-the-job trainee & $16(38.1)$ \\
Board certification of on-board physicians ${ }^{\mathrm{b}}$ & $N=347$ \\
Emergency medicine & $263(75.8)$ \\
General surgery & $54(15.6)$ \\
Intensive care & $52(15.0)$ \\
Anesthesiology & $35(10.1)$ \\
Cranial surgery & $16(4.6)$ \\
Orthopedics & $14(4.0)$ \\
Cardiovascular medicine & $14(4.0)$ \\
Respiratory medicine & $4(1.2)$ \\
Any other board certifications & $55(15.9)$ \\
Certification of on-board nurses & b \\
Emergency nursing & $N=326$ \\
Intensive care & $58(17.8)$ \\
Pediatric emergency nursing & $3(0.9)$ \\
Any other certifications & $1(0.3)$ \\
\hline
\end{tabular}

${ }^{a}$ Based on the replies of 42 of the 45 HEMS queried

b Physicians and nurses may have more than one on-board certification the prehospital setting, except in immediate life-threatening scenarios (i.e., airway obstruction). Otherwise, to avoid a potentially catastrophic situation, oxygen should be optimized and hospital transfer accelerated to obtain prompt access to advanced in-hospital human and equipment resources [10].

\section{Limitations in the call for help in prehospital settings}

More than $85 \%$ of the bases in this study had no back-up personnel. The 'call for help' is the first step and the most important aspect of the DAM algorithms [11-13]. Jaber et al. [27] recently reported that having two care providers present was a vital element for successful ETI of critically ill patients. Limited help is one of the greatest disadvantages of HEMS and the situation most unlike that of a hospital. DAM in the poorly prepared prehospital setting can lead to serious adverse events regarding patient care. Past reports from outside Japan have shown increases in ETI difficulty [3-6] and severe ETI-related complications [28, 29] when the procedure is performed outside hospitals. In fact, under these high-risk conditions ETI should not be attempted if manual ventilation is successful. Paal et al. [10] also emphasized the importance of avoiding repeat ETI attempts in prehospital settings. According to the best available evidence, prehospital ETI does not provide any survival
Fig. 2 Availability in Japanese helicopter emergency medical services of the difficult airway management resources specified in the JSA, ASA, and DAS airway management algorithms. ASA American Society of Anesthesiologists, DAS Difficult Airway Society, JSA Japanese Society of Anesthesiologists, $S G A$ supraglottic airway device

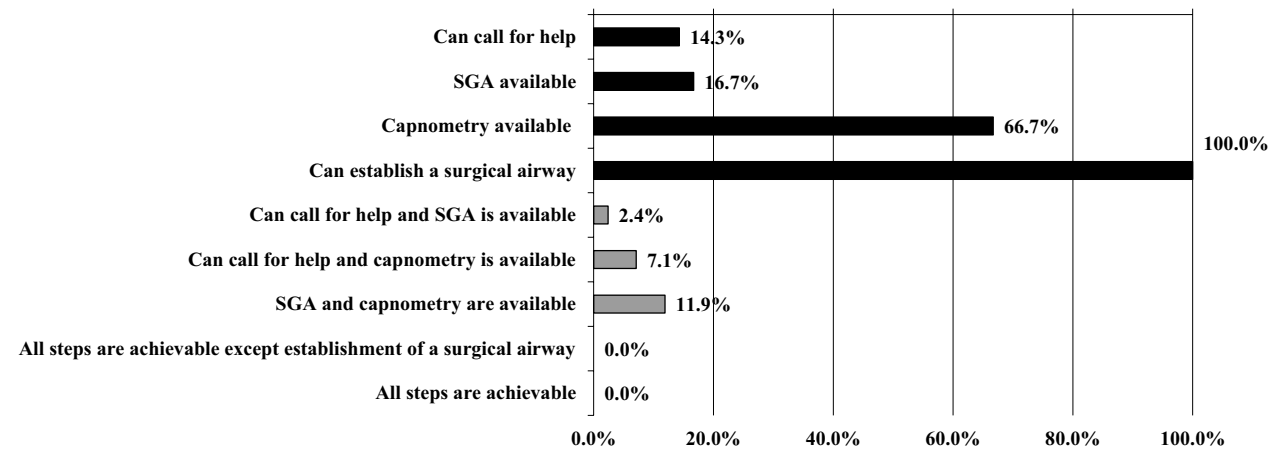

Table 4 Association between the availability of difficult airway management resources specified in the JSA, ASA, and DAS airway management algorithms and the number of dispatches, region, and foundation date in Japanese helicopter emergency medical services (HEMS)

\begin{tabular}{|c|c|c|c|c|c|c|c|c|c|}
\hline & \multicolumn{3}{|c|}{ Number of dispatches (year) } & \multicolumn{3}{|l|}{ Region } & \multicolumn{3}{|l|}{ Foundation date } \\
\hline & \multicolumn{3}{|l|}{$N(\%)$} & \multicolumn{3}{|l|}{$N(\%)$} & \multicolumn{3}{|l|}{$N(\%)$} \\
\hline & $\geq 447 N=21$ & $<447 N=21$ & $p^{\mathrm{a}}$ & East $N=22$ & West $N=20$ & $p^{\mathrm{a}}$ & $2001-2009 N=19$ & $2010-2015 N=23$ & $p^{\mathrm{a}}$ \\
\hline Can call for help & $3(14.3)$ & $3(14.3)$ & 1.00 & $4(18.2)$ & $2(10.0)$ & 0.67 & $2(10.5)$ & $4(17.4)$ & 0.67 \\
\hline $\begin{array}{c}\text { Supraglottic airway } \\
\text { device available }\end{array}$ & $2(9.5)$ & $5(23.8)$ & 0.41 & $5(22.7)$ & $2(10.0)$ & 0.41 & $3(15.8)$ & $4(17.4)$ & 1.00 \\
\hline Capnometry available & $14(66.7)$ & $14(66.7)$ & 1.00 & $17(77.3)$ & $11(55.0)$ & 0.19 & $13(68.4)$ & $15(65.2)$ & 1.00 \\
\hline
\end{tabular}

Based on the replies of 42 of the 45 HEMS queried

ASA American Society of Anesthesiologists, DAS Difficult Airway Society, JSA Japanese Society of Anesthesiologists

${ }^{a} p$ values derived from Fisher's exact test 
benefits for patients suffering out-of-hospital cardiac arrest $[30,31]$, traumatic brain injury [32-34], or multiple traumas [35-37]. These patients are the most vulnerable to the detrimental cardiovascular effects of the positive pressure breaths delivered through an endotracheal tube [38]. Davis et al. [32] showed that, even after adjusting for multiple clinical variables affecting outcome, prehospital intubation was associated with decreased survival among patients with moderate to severe traumatic brain injury. Warner et al. [34] found a correlation between patients with severe traumatic brain injury who received prehospital rapid sequence intubation (RSI) and both mortality and hyperventilation (arterial $\mathrm{PCO}_{2}<24 \mathrm{mmHg}$ ). Shafi et al. [35] demonstrated that prehospital ETI in trauma patients is associated with decreased survival, possibly because of positive pressure ventilation during the hypovolemic state. Stockinger et al. [36] found that prehospital ETI conferred no survival benefit over bag valve mask ventilation and increased prehospital time. These studies, together with our own, suggest that the threshold for ETI outside the hospital should be higher than in the hospital emergency room. At least in Japan, if not elsewhere, the evidence underlines the need for a deliberation of the risks and benefits of prehospital ETI.

\section{Neglect of the importance of SGA as a rescue ventilation device in prehospital settings in Japan}

In this study, SGA was available in only $16.7 \%$ of the prehospital settings, and a pediatric-sized device in only $11.9 \%$. In Europe, SGA is available in $92.0-97.6 \%$ of prehospital settings $[19,20]$. Thus, in Japan, SGA has been undervalued as a rescue ventilation device in prehospital settings. Each HEMS must have back-up ventilation strategies [11-13] because the consequences of failed intubation can be devastating. SGAs have several advantages for use in rescue ventilation [11-13] and should be available wherever anesthesia is carried out in the prehospital setting $[15,16]$. Lockey et al. [39] and Combes et al. [40] reported that all patients whose tracheas could not be intubated in a prehospital emergency setting were successfully rescued by SGA. Our study showed that prehospital airway equipment was arbitrarily selected by each base. However, its standardization, including a SGA or other rescue ventilator device would be beneficial.

Successful SGA insertion is related to operator experience [41]. In a CVCI scenario, which is a definite opportunity to use SGA [11-13], the victim is at high risk of cardiac arrest due to hypoxemia. Therefore, appropriate training in SGA insertion is crucial for health care professionals who are likely to participate in airway management. Nevertheless, other than elective surgery, the clinical settings in which patients are ventilated with SGA are relatively rare. To gain SGA insertion experience and airway management competence, HEMS physicians should participate in a certain number of these procedures in the hospital operating room [42, 43]; this is especially important for those whose clinical background is not anesthesiology. Thus, as in other countries [42, 43], airway management training programs in the operating room for HEMS physicians should be established throughout Japan.

\section{Limited availability of capnometry in prehospital settings in Japan}

Capnometry was available in approximately two-thirds of the HEMS bases surveyed. By contrast, capnometry is available in $85-100 \%$ of the prehospital settings in Europe $[19,20]$. Verification of endotracheal tube placement is an indispensable part of any DAM strategy [1113], and capnometry is both more sensitive and more specific than auscultation alone in recognizing correct tube placement following emergency intubation [44-46]. Continuous end-tidal carbon dioxide $\left(\mathrm{ETCO}_{2}\right)$ monitoring is also useful to detect inadvertent tube dislodgement during patient transport [47]. Silvestri et al. [47] reported that when paramedics used continuous $\mathrm{ETCO}_{2}$ monitoring in prehospital settings, there were no cases of unrecognized misplaced intubation in patients upon emergency room arrival, whereas the misplaced intubation rate was $23 \%$ when continuous $\mathrm{ETCO}_{2}$ was not used. A national audit in the UK [14] found that failure to use capnometry in treating a difficult airway probably contributed to at least some of the fatal outcomes. $\mathrm{ETCO}_{2}$ confirmation of tube placement and continuous monitoring of the endotracheal tube position are now a standard of care in the operating room [11-13] and in the intensive care unit [14]. As a result, the use of $\mathrm{ETCO}_{2}$ monitoring has become an important aspect of emergency medicine [14, 47]. The incorporation of $\mathrm{ETCO}_{2}$ confirmation and continuous monitoring into out-of-hospital airway management would therefore improve patient management by prehospital health care professionals.

\section{Shortage of board-certified anesthesiologists as prehospital physicians in Japan}

According to our survey, board-certified anesthesiologists comprised only $10.1 \%$ of all prehospital physicians in Japan. In Scandinavia and Germany, by contrast, prehospital airway management is mostly performed by anesthesiologists with specific prehospital training [15, 17, 48, 49]. As concluded by Lockey et al. [15] and clearly stated in the prehospital advanced airway guidelines of Scandinavia [17], the providers of prehospital airway management should have the same level of competence as in-hospital anesthesia providers. To date, standard airway management 
competence for HEMS physicians in Japan has not been defined. Breckwoldt et al. [6] investigated the incidence of difficult ETI (number of ETI attempts $>3$ ) in the prehospital setting, comparing emergency physicians with a clinical background in anesthesiology (expert status) and those with a background in internal medicine. They found an association between expert status and a significantly lower incidence of difficult ETI and thus proposed that the value of day-to-day ETI experience be considered in the treatment of a difficult airway outside the hospital. As we pointed out in a previous study, the skill and knowledge of anesthesiologists should be fully employed for high-risk ETI rather than limited to the operating room [50]. To improve prehospital airway management in Japan, more anesthesiologists are recommended to participate in prehospital medical care. There is however a lack of anesthesiologists in Japan, and the regular training of HEMS (non-anesthesiologist) physicians in the operating room would also be beneficial for airway training and to gain experience [42, 43]. For the retention of ETI skills, HEMS physicians should be required to perform a certain number of procedures within a defined period [51].

\section{Preparedness of HEMS to perform surgical airway management}

While all bases had surgical airway devices, few had reversal agents. This finding probably reflects the fact that in the field of emergency medicine, even if difficulties are encountered, waking a patient following RSI is rare [39, 52], because a patient requiring emergency ETI is absolutely in need of a definitive airway. In these settings, a timely surgical airway may be life-saving [53] and more important than waking the patient. Previous studies reported an incidence of prehospital cricothyroidotomy of $0.5-2.4 \%$ [39, 54, 55], compared with $0.005-0.025 \%$ [56] in the operating room. The need for an emergency surgical procedure was 100 -fold higher in prehospital settings than in the hospital operating room. All HEMS physicians therefore must be proficient in this alternative intubation technique. To maintain their proficiency, they should receive regular off-the-job training in, for example, the use of a simulator [57, 58].

\section{Recommendations from this study}

This study revealed that the limited availability of backup personnel, alternative ventilation, and confirmation equipment in prehospital settings in Japan greatly hinders DAM. Given the current situation in Japan, rapid transport is preferable over active airway management in the field if ventilation and oxygenation are acceptable. Avoiding a prehospital ETI attempt is particularly important if a difficult airway is anticipated [10]. As stated by the Scandinavian
Society for Anaesthesiology and Intensive Care Medicine [17], "Even for maximally skilled personnel, it should always be considered whether ETI attempts should be performed pre-hospitally or be postponed till more advanced in-hospital techniques are available." Nevertheless, 'forced to act' scenarios may arise despite an anticipated difficult airway [59]. Examples include a patient with immediate or deteriorating airway obstruction or a patient whose oxygenation is unacceptable even after manual bag mask ventilation. In these cases, multiple ETI attempts should be strictly prohibited and a rescue technique, including a surgical airway, should be performed without hesitation because (1) multiple ETI attempts in a setting of limited human and equipment resources are known to increase the risk of severe complications $[7-9,14,28,29]$ and (2) complications in the management of a difficult airway can increase prehospital time, which is associated with an adverse outcome [60-62]. Thus, in prehospital settings, a difficult airway should be managed in a time-sensitive manner [59]. There is a tendency for laryngoscopists to persist with an ETI even if it is proving to be difficult [63]; this inevitably results in the delayed implementation of alternative intubation techniques. However, any hesitancy regarding the latter will be readily overcome once proficiency with an alternative rescue technique is acquired [57].

In Japan, prehospital airway equipment is not standardized; it is selected at the discretion of the manager of each base. To ensure homogeneous prehospital airway strategies, the equipment carried out-of-hospital needs to be standardized and should be consistent with that of a hospital operating room [14]. Suggestions for DAM resources have been proposed by the JSA [11], ASA [12], and DAS [13] which include rigid laryngoscope blades of alternate design and size from those routinely used, video laryngoscope, tracheal tubes of assorted sizes, tracheal tube guides including a stylet and a gum elastic bougie, noninvasive airway ventilation equipment including assorted sizes of SGA and nasal/oral airway, equipment suitable for emergency invasive airway access, an exhaled carbon dioxide detector, and a portable storage unit containing these devices.

Adequate experience and the training of every HEMS physician in the use of this equipment are absolute requirements. Airway management training programs for HEMS physicians [42, 43] that include sufficient ETI and SGA caseloads in the operating room should be available throughout Japan. Regular off-the-job training can aid in maintaining the skills needed for surgical airway management $[57,58]$.

\section{Study limitations and advantages}

There were two major limitations to this study. First, our survey did not determine the frequency of difficult airways 
and CVCI situations, nor did it obtain information on airway management practices in prehospital settings. The optimal management of difficult airway situations despite the limited resources of HEMS bases remains to be determined in future works. Second, because our questionnaire was self-administered, there may have been reporting bias. Nonetheless, in our survey of HEMS hospitals in Japan, the response rate was extremely high (42 of 45). Our study thus provides an accurate depiction of the current state of prehospital advanced airway management in Japan but it also reveals the areas in need of improvement.

\section{Conclusion}

In Japan, compliance with standard airway management algorithms is currently not practicable [11-13] in prehospital settings, given the limited availability of alternative ventilation equipment and back-up personnel. Because the prehospital setting in Japan is not conducive to successful DAM, all healthcare professionals working in this environment should seriously consider whether ETI should be performed or whether the more prudent decision is to postpone the procedure until more advanced in-hospital techniques and an adequate number of personnel are available. In addition, the airway equipment, alternative ventilation equipment, and confirmation device carried out of hospital should be standardized. Because adequate experience is essential in the successful management of challenging situations, airway management training programs for HEMS physicians should be made available throughout Japan.

Acknowledgments We thank all of the participating HEMS bases in Japan for their earnest and generous cooperation in this project. We also thank the Japanese Society for Aeromedical Services and HEM-Net for providing essential data on the trend in annual HEMS dispatches and the number of HEMS bases in Japan. The authors are grateful to the anonymous reviewers for their valuable suggestions, which greatly improved the scientific merit of the paper. Finally, we thank Nozomi Ono, M.D. (Department of Psychiatry, Hoshigaoka Hospital, Koriyama, Japan), for her assistance in reviewing the manuscript.

\section{Compliance with ethical standards}

Conflict of interest The authors have no competing interests to declare.

Open Access This article is distributed under the terms of the Creative Commons Attribution 4.0 International License (http://creativecommons.org/licenses/by/4.0/), which permits unrestricted use, distribution, and reproduction in any medium, provided you give appropriate credit to the original author(s) and the source, provide a link to the Creative Commons license, and indicate if changes were made.

\section{References}

1. Nishikawa W, Yamano Y. An overview of the development of helicopter emergency medical services in Japan. Air Med J. 2010;29:288-91.

2. Matsumoto H, Motomura T, Hara Y, Masuda Y, Mashiko K, Yokota H, Koido Y. Lessons learned from the aeromedical disaster relief activities following the great East Japan earthquake. Prehosp Disaster Med. 2013;28:166-9.

3. Combes X, Jabre P, Margenet A, Merle JC, Leroux B, Dru M, Lecarpentier E, Dhonneur G. Unanticipated difficult airway management in the prehospital emergency setting: prospective validation of an algorithm. Anesthesiology. 2011;114:105-10.

4. Adnet F, Borron SW, Racine SX, Clemessy JL, Fournier JL, Plaisance P, Lapandry C. The intubation difficulty scale (IDS): proposal and evaluation of a new score characterizing the complexity of endotracheal intubation. Anesthesiology. 1997;87:1290-7.

5. Timmermann A, Eich C, Russo SG, Natge U, Brauer A, Rosenblatt WH, Braun U. Prehospital airway management: a prospective evaluation of anaesthesia trained emergency physicians. Resuscitation. 2006;70:179-85.

6. Breckwoldt J, Klemstein S, Brunne B, Schnitzer L, Arntz HR, Mochmann HC. Expertise in prehospital endotracheal intubation by emergency medicine physicians? Comparing 'proficient performers' and 'experts'. Resuscitation. 2012;83:434-9.

7. Mort TC. Emergency tracheal intubation: complications associated with repeated laryngoscopic attempts. Anesth Analg. 2004;99:607-13.

8. Hasegawa K, Shigemitsu K, Hagiwara Y, Chiba T, Watase H, Brown CA 3rd, Brown DF, Japanese Emergency Medicine Research Alliance Investigators. Association between repeated intubation attempts and adverse events in emergency departments: an analysis of a multicenter prospective observational study. Ann Emerg Med. 2012;60:749-54.

9. Martin LD, Mhyre JM, Shanks AM, Tremper KK, Kheterpal S. 3423 emergency tracheal intubations at a university hospital: airway outcomes and complications. Anesthesiology. 2011;114:42-8.

10. Paal P, Herff H, Mitterlechner T, von Goedecke A, Brugger H, Lindner KH, Wenzel V. Anaesthesia in prehospital emergencies and in the emergency room. Resuscitation. 2010;81:148-54.

11. Japanese Society of Anesthesiologists. JSA airway management guideline 2014: to improve the safety of induction of anesthesia. J Anesth. 2014;28:482-93.

12. Apfelbaum JL, Hagberg CA, Caplan RA, Blitt CD, Connis RT, Nickinovich DG, Hagberg CA, Caplan RA, Benumof JL, Berry FA, Blitt CD, Bode RH, Cheney FW, Connis RT, Guidry OF, Nickinovich DG, Ovassapian A. American Society of Anesthesiologists Task Force on Management of the Difficult Airway. Practice guidelines for management of the difficult airway: an updated report by the American Society of Anesthesiologists Task Force on Management of the Difficult Airway. Anesthesiology. 2013;118:251-70.

13. Henderson JJ, Popat MT, Latto IP, Pearce AC, Difficult Airway Society. Difficult Airway Society guidelines for management of the unanticipated difficult intubation. Anaesthesia. 2004;59:675-94.

14. Cook TM, Woodall N, Harper J, Benger J, Fourth National Audit Project. Major complications of airway management in the UK: results of the Fourth National Audit Project of the Royal College of Anaesthetists and the Difficult Airway Society. Part 2: Intensive care and emergency departments. $\mathrm{Br} \mathrm{J}$ Anaesth. 2011;106:632-42. 
15. Lockey DJ, Crewdson K, Lossius HM. Pre-hospital anaesthesia: the same but different. Br J Anaesth. 2014;113:211-9.

16. Lockey D, Porter K, University Hospital Birmingham Faculty of Pre-hospital Care. Prehospital anaesthesia in the UK: position statement on behalf of the Faculty of Pre-hospital Care. Emerg Med J. 2007;24:606-7.

17. Berlac P, Hyldmo PK, Kongstad P, Kurola J, Nakstad AR, Sandberg M, Scandinavian Society for Anesthesiology and Intensive Care Medicine. Pre-hospital airway management: guidelines from a task force from the Scandinavian Society for Anaesthesiology and Intensive Care Medicine. Acta Anaesthesiol Scand. 2008;52:897-907.

18. Rognas LK, Hansen TM. EMS-physicians' self reported airway management training and expertise; a descriptive study from the Central Region of Denmark. Scand J Trauma Resusc Emerg Med. 2011;19:10.

19. Schmid M, Mang H, Ey K, Schuttler J. Prehospital airway management on rescue helicopters in the United Kingdom. Anaesthesia. 2009;64:625-31.

20. Schmid M, Schuttler J, Ey K, Reichenbach M, Trimmel H, Mang H. Equipment for pre-hospital airway management on Helicopter Emergency Medical System helicopters in central Europe. Acta Anaesthesiol Scand. 2011;55:583-7.

21. Morton T, Brady S, Clancy M. Difficult airway equipment in English emergency departments. Anaesthesia. 2000;55:485-8.

22. Levitan RM, Kush S, Hollander JE. Devices for difficult airway management in academic emergency departments: results of a national survey. Ann Emerg Med. 1999;33:694-8.

23. Walsh K, Cummins F. Difficult airway equipment in departments of emergency medicine in Ireland: results of a national survey. Eur J Anaesthesiol. 2004;21:128-31.

24. Niazi A, Cummins E, Walsh K. Difficult airway equipment in obstetric units in the republic of Ireland: results of a national survey. Eur J Anaesthesiol. 2004;21:861-3.

25. Stamer UM, Messerschmidt A, Wulf H, Hoeft A. Equipment for the difficult airway in obstetric units in Germany. J Clin Anesth. 2000;12:151-6.

26. Bullough AS, Carraretto M. A United Kingdom national obstetric intubation equipment survey. Int $\mathrm{J}$ Obstet Anesth. 2009; 18:342-5.

27. Jaber S, Jung B, Corne P, Sebbane M, Muller L, Chanques G, Verzilli D, Jonquet O, Eledjam JJ, Lefrant JY. An intervention to decrease complications related to endotracheal intubation in the intensive care unit: a prospective, multiple-center study. Intensive Care Med. 2010;36:248-55.

28. Timmermann A, Russo SG, Eich C, Roessler M, Braun U, Rosenblatt WH, Quintel M. The out-of-hospital esophageal and endobronchial intubations performed by emergency physicians. Anesth Analg. 2007;104:619-23.

29. Ufberg JW, Bushra JS, Karras DJ, Satz WA, Kueppers F. Aspiration of gastric contents: association with prehospital intubation. Am J Emerg Med. 2005;23:379-82.

30. Studnek JR, Thestrup L, Vandeventer S, Ward SR, Staley K, Garvey L, Blackwell T. The association between prehospital endotracheal intubation attempts and survival to hospital discharge among out-of-hospital cardiac arrest patients. Acad Emerg Med. 2010;17:918-25.

31. Hasegawa K, Hiraide A, Chang Y, Brown DF. Association of prehospital advanced airway management with neurologic outcome and survival in patients with out-of-hospital cardiac arrest. JAMA. 2013;16(309):257-66.

32. Davis DP, Peay J, Sise MJ, Vilke GM, Kennedy F, Eastman AB, Velky T, Hoyt DB. The impact of prehospital endotracheal intubation on outcome in moderate to severe traumatic brain injury. $\mathrm{J}$ Trauma. 2005;58:933-9.
33. von Elm E, Schoettker P, Henzi I, Osterwalder J, Walder B. Pre-hospital tracheal intubation in patients with traumatic brain injury: systematic review of current evidence. $\mathrm{Br} \mathrm{J}$ Anaesth. 2009;103:371-86

34. Warner KJ, Cuschieri J, Copass MK, Jurkovich GJ, Bulger EM. The impact of prehospital ventilation on outcome after severe traumatic brain injury. J Trauma. 2007;62:1330-6.

35. Shafi S, Gentilello L. Pre-hospital endotracheal intubation and positive pressure ventilation is associated with hypotension and decreased survival in hypovolemic trauma patients: an analysis of the National Trauma Data Bank. J Trauma. 2005;59:1140-5.

36. Stockinger ZT, McSwain NE Jr. Prehospital endotracheal intubation for trauma does not improve survival over bag-valve-mask ventilation. J Trauma. 2004;56:531-6.

37. Kempema J, Trust MD, Ali S, Cabanas JG, Hinchey PR, Brown LH, Brown CV. Prehospital endotracheal intubation vs extraglottic airway device in blunt trauma. Am J Emerg Med. 2015;33:1080-3.

38. Pepe PE, Roppolo LP, Fowler RL. Prehospital endotracheal intubation: Elemental or detrimental? Crit Care. 2015;19:121.

39. Lockey D, Crewdson K, Weaver A, Davies G. Observational study of the success rates of intubation and failed intubation airway rescue techniques in 7256 attempted intubations of trauma patients by pre-hospital physicians. Br J Anaesth. 2014;113:220-5.

40. Combes X, Jabre P, Margenet A, Merle JC, Leroux B, Dru M, Lecarpentier E, Dhonneur G. Unanticipated difficult airway management in the prehospital emergency setting: prospective validation of an algorithm. Anesthesiology. 2011;114:105-10.

41. McCrirrick A, Ramage DT, Pracilio JA, Hickman JA. Experience with the laryngeal mask airway in two hundred patients. Anaesth Intensive Care. 1991;19:256-60.

42. Sollid SJ, Heltne JK, Soreide E, Lossius HM. Pre-hospital advanced airway management by anaesthesiologists: Is there still room for improvement? Scand J Trauma Resusc Emerg Med. 2008;16:2.

43. Davis DP, Buono C, Ford J, Paulson L, Koenig W, Carrison D. The effectiveness of a novel, algorithm-based difficult airway curriculum for air medical crews using human patient simulators. Prehosp Emerg Care. 2007;11:72-9.

44. Takeda T, Tanigawa K, Tanaka H, Hayashi Y, Goto E, Tanaka K. The assessment of three methods to verify tracheal tube placement in the emergency setting. Resuscitation. 2003;56:153-7.

45. Grmec S. Comparison of three different methods to confirm tracheal tube placement in emergency intubation. Intensive Care Med. 2002;28:701-4.

46. Grmec S, Mally S. Prehospital determination of tracheal tube placement in severe head injury. Emerg Med J. 2004;21:518-20.

47. Silvestri S, Ralls GA, Krauss B, Thundiyil J, Rothrock SG, Senn A, Carter E, Falk J. The effectiveness of out-of-hospital use of continuous end-tidal carbon dioxide monitoring on the rate of unrecognized misplaced intubation within a regional emergency medical services system. Ann Emerg Med. 2005;45:497-503.

48. Helm M, Hossfeld B, Schafer S, Hoitz J, Lampl L. Factors influencing emergency intubation in the pre-hospital setting-a multicentre study in the German Helicopter Emergency Medical Service. Br J Anaesth. 2006;96:67-71.

49. Sollid SJ, Lossius HM, Soreide E. Pre-hospital intubation by anaesthesiologists in patients with severe trauma: an audit of a Norwegian helicopter emergency medical service. Scand J Trauma Resusc Emerg Med. 2010;18:30.

50. Ono Y, Kikuchi H, Hashimoto K, Sasaki T, Ishii J, Tase C, Shinohara K. Emergency endotracheal intubation-related adverse events in bronchial asthma exacerbation: Can anesthesiologists attenuate the risk? J Anesth. 2015;29:678-85. 
51. Konrad C, Schupfer G, Wietlisbach M, Gerber H. Learning manual skills in anesthesiology: Is there a recommended number of cases for anesthetic procedures? Anesth Analg. 1998;86:635-9.

52. Lyon RM, Perkins ZB, Chatterjee D, Lockey DJ, Russell MQ, Kent, Surrey \& Sussex Air Ambulance Trust. Significant modification of traditional rapid sequence induction improves safety and effectiveness of pre-hospital trauma anaesthesia. Crit Care. 2015;19:134.

53. Ono Y, Yokoyama H, Matsumoto A, Kumada Y, Shinohara K, Tase C. Surgical airways for trauma patients in an emergency surgical setting: 11 years' experience at a teaching hospital in Japan. J Anesth. 2013;27:832-7.

54. Robinson KJ, Katz R, Jacobs LM. A 12-year experience with prehospital cricothyrotomies. Air Med J. 2001;20:27-30.

55. McIntosh SE, Swanson ER, Barton ED. Cricothyrotomy in air medical transport. J Trauma. 2008;64:1543-7.

56. Berkow LC, Greenberg RS, Kan KH, Colantuoni E, Mark LJ, Flint PW, Corridore M, Bhatti N, Heitmiller ES. Need for emergency surgical airway reduced by a comprehensive difficult airway program. Anesth Analg. 2009;109:1860-9.

57. Vissers RJ, Bair AE. Surgical airway management. In: Walls RM, Murphy MF, editors. Manual of emergency airway management. 4th ed. Philadelphia: Lippincott Williams \& Wilkins; 2012. p. 193-219.

58. Wong DT, Prabhu AJ, Coloma M, Imasogie N, Chung FF. What is the minimum training required for successful cricothyroidotomy? A study in Mannequins. Anesthesiology. 2003;98:349-53.

59. Eichel JL, Skarote MB, Murphy MF. Difficult and failed airway management in EMS. In: Walls RM, Murphy MF, editors. Manual of emergency airway management. 4th ed. Philadelphia: Lippincott Williams \& Wilkins; 2012. p. 340-6.

60. Feero S, Hedges JR, Simmons E, Irwin L. Does out-of-hospital EMS time affect trauma survival? Am J Emerg Med. 1995;13:133-5.

61. Birk HO, Henriksen LO. Prehospital interventions: on-scenetime and ambulance-technicians' experience. Prehosp Disaster Med. 2002;17:167-9.

62. Gonzalez RP, Cummings G, Mulekar M, Rodning CB. Increased mortality in rural vehicular trauma: identifying contributing factors through data linkage. J Trauma. 2006;61:404-9.

63. Cook TM, MacDougall-Davis SR. Complications and failure of airway management. Br J Anaesth. 2012;109(Suppl 1):i68-85. 